\title{
O Tratamento Familiar em Casos de Dependência de Drogas no Brasil: Revisão de Literatura
}

\author{
Ana Paula Magosso Cavaggioni* \\ Miria Benincasa Gomes** \\ Manuel Morgado Rezende***
}

\begin{abstract}
Resumo
A dependência de drogas constitui sério problema de saúde pública, necessitando do desenvolvimento de estratégias tanto para tratamento como promoção e prevenção de saúde de usuários e familiares. Este artigo trata-se de uma revisão da literatura sobre a produção científica acerca dos tratamentos oferecidos aos familiares de dependentes químicos no Brasil, ao longo dos últimos 10 anos através da biblioteca virtual da Bireme. Observa-se escassa produção de conhecimento científico visando o desenvolvimento de estratégias de tratamento familiar viáveis, acessíveis e que não se limitem apenas a espaços grupais informativos, educativos e de acolhimento, demonstrando a necessidade do desenvolvimento de estratégias que impactem em aspectos subjetivos subjacentes, latentes, que permeiam a constituição das relações familiares, a manutenção e repetição do sintoma.

Palavras-chave: Transtornos Relacionados ao Uso de Substâncias; Medicina de Família e Comunidade; Promoção de Saúde.
\end{abstract}

\section{The familiar treatment in drug addiction cases in Brazil : literature review}

\begin{abstract}
Drug addiction is a serious public health problem, requiring the development of treatment and prevention/promotion of health for pacients and their families. This article is a literature review of the scientific literature about the treatments offered to the families of drug addicts in Brazil over the past 10 years through the Virtual Library Bireme. It is observed scarce production of scientific knowledge for the development of viable and accessible family treatment strategies, that are not limited only to informative groups, educational and emotional shelter spaces, demonstrating the need to develop strategies that impact on underlying latent subjective aspects that permeate the creation of family relations, the maintenance and repetition of the symptoms.
\end{abstract}

Keywords: Substance-Related Disorders; Family Practice; Health Promotion.

* Psicóloga, mestranda pelo curso de Pós-Graduação em Psicologia da Saúde pela UMESP

** Doutora em Psicologia do Desenvolvimento pela USP, Docente do PPGPS da Metodista.

*** Doutor em Saúde Mental pela Unicamp, Docente do PPGPS da Metodista 


\section{Introdução}

A dependência química há tempos tornou-se problema de saúde pública. Segundo Relatório Mundial sobre Drogas (ONU, 2015), a prevalência mundial de uso de drogas continua estável. Estima-se que cerca de 5\% da população mundial, ou seja, em torno de 246 milhões de pessoas, entre 15 e 64 anos de idade utilizaram drogas ilícitas em 2013, apresentando um importante impacto na saúde. Destas, em torno de 27 milhões são dependentes químicos, sendo que metade deste número se refere ao uso de drogas injetáveis. Porém, apesar da estabilidade na prevalência do uso de drogas, apenas $1 / 6$ das pessoas tem acesso ao tratamento. O Relatório Mundial sobre Drogas indica que o índice de mortes entre usuários dependentes de drogas injetáveis é 15 vezes superior ao restante da população.

No Brasil é elevado o número de pessoas que consomem de forma abusiva substâncias psicoativas (Aguilar \& Pillon, 2005; Canoletti, 2005). Uma pesquisa brasileira realizada em 149 municípios do país revela que o país se encontra entre os maiores consumidores de cocaína. Estima-se que, em 2011, cerca de 4\% da população brasileira tivesse experimentado a droga pelo menos uma vez na vida, o que significa mais de 52 milhões de pessoas. E destes, 15,6\% tornaram-se dependentes químicos (Abdalla, Madruga, Ribeiro, Pinsky, Caetano \& Laranjeira, 2014).

Porém, segundo dados do Ministério da Saúde (2007), apesar da atenção que passou a ser dada à saúde mental após a Reforma Psiquiátrica no Brasil, que só pode consolidar-se em 2001, com a aprovação da Lei Federal 10.216, a saúde pública manteve-se omissa em relação às necessidades de prevenção e tratamento de transtornos associados ao uso de álcool e outras drogas, deixando-a a cargo da segurança pública, da pedagogia e de associações religiosas. Apenas em 2002, em consonância com as recomendações da III Conferência Nacional de Saúde Mental, o Ministério da Saúde instituiu, no âmbito do Sistema Único de Saúde (SUS), o Programa Nacional de Atenção Comunitária Integrada aos Usuários de Álcool e Outras Drogas. A partir daí a dependência química passou a ser reconhecida como uma questão de saúde pública, inserida no campo da saúde mental.

O Programa Nacional de Atenção Comunitária Integrada aos Usuários de Álcool e Outras Drogas passou a organizar ações de promoção, prevenção, proteção à saúde e educação dos usuários, além de estabelecer uma rede de serviços extra-hospitalares articuladas à rede de atenção psicossocial, fundamentada na estratégia de redução de danos. Neste contexto, surgiu o CAPS-AD - Centro de Atenção Psicossocial para atendimento de pacientes com dependência e/ou uso prejudicial de Álcool e outras Drogas - que deve, ativa e sistematicamente, buscar as necessidades da população a partir do meio cultural e comunitário na qual estão inseridos (Ministério da Saúde, 2004).

O Observatório Brasileiro de Informações sobre Drogas (OBID), desenvolvido pela Secretaria Nacional de Políticas sobre Drogas, em 2002 durante a Semana Nacional Antidrogas, com apoio financeiro do Ministério da Saúde, reúne, coordena e mantém atualizado o conhecimento disponível sobre o assunto, buscando fundamentar o desenvolvimento de programas e intervenções voltadas a redução de demanda e oferta de drogas. Quando abordam os princípios de tratamento efetivo, fica claro, dentre outros aspectos, a inexistência de um tratamento único, da necessidade do acesso ao tratamento pelo usuário e de tratamentos outros além da psicoterapia individual, como a terapia familiar (Rezende, 2001). A política sobre Drogas do Ministério da Justiça do Governo Federal deixa clara a importância da família durante todo o processo de tratamento do dependente químico. Segundo esta, a família é fundamental não apenas durante o tratamento, mas também para evitar as chances de recaída e melhorar a qualidade de vida do paciente. Para isso, é importante que ela esteja implicada com o tratamento e tenha condições de acolher o dependente químico e manter um ambiente familiar afetivo positivo (Ministério da Saúde, 20107).

Rezende (2001) concorda que a intervenção familiar se trata de uma estratégia importante e relevante no tratamento da dependência química, devendo ser incluída no programa de tratamento do dependente químico. Porém, para que um familiar possa estar verdadeiramente implicado no tratamento de forma a colaborar com o mesmo, é fundamental que busque tratamento especializado, pois a dependência química não apenas é reveladora da dinâmica familiar como também impacta nas relações estabelecidas entre seus membros (Kalina, 1988).

Este artigo consiste justamente em, através de uma revisão de literatura, verificar as estratégias de tratamento de familiares de dependentes químicos que vem sendo disponibilizadas no Brasil na última década.

\section{Método}

Neste artigo apresenta-se uma revisão da literatura sobre a produção científica acerca dos tratamentos oferecidos aos familiares de dependentes químicos no 
Brasil, ao longo dos últimos 10 anos. Foi realizada uma pesquisa bibliográfica consultando as bases de dados MEDLINE, Lilacs, BDENF - enfermagem, através da biblioteca virtual da Bireme, com a utilização das seguintes palavras-chave: "toxicomania" "tratamento familiar" e "Brasil" (abrangendo título, resumo e assunto), considerando o período de 2005 a 2015. Os artigos encontrados foram categorizados, sendo excluídos aqueles que não se referiam a intervenções familiares implementadas no Brasil.

\section{Resultados}

Os artigos encontrados constam no quadro 1:

\section{Resultados}

Quadro 1 - Revisão da Literatura

\begin{tabular}{|c|c|c|c|c|c|}
\hline & Titulo & Autor & Base de dados & Ano & Área \\
\hline 1 & $\begin{array}{l}\text { Terapia comunitária integrativa: situações de sofri- } \\
\text { mento emocional e estratégias de enfrentamento } \\
\text { apresentadas por usuários / Terapia comunitaria } \\
\text { integradora: situaciones de angustia emocional y } \\
\text { estrategias de afrontamiento presentadas por losusu- } \\
\text { arios / Communityintegrativetherapy: situations of } \\
\text { emotionalsufferingandpatients' copingstrategies }\end{array}$ & $\begin{array}{l}\text { Rocha, Ianine Alves da; Sá, Ara- } \\
\text { linda Nogueira Pinto de; Braga, } \\
\text { Lucineide Alves Vieira; Ferreira } \\
\text { Filha, Maria de Oliveira;Dias, } \\
\text { Maria Djair. }\end{array}$ & $\begin{array}{l}\text { BDENF-En- } \\
\text { fermagem }\end{array}$ & 2013 & Enfermagem \\
\hline 3 & $\begin{array}{l}\text { Factores de la satisfacciónsobre el apoyo familiar } \\
\text { del adolescenteadictoentratamiento / Factors about } \\
\text { the family support of the addicted teenager in treat- } \\
\text { ment }\end{array}$ & $\begin{array}{l}\text { Velásquez Carranza, Doris; Vai- } \\
\text { zBonifaz, Rosa; Pedrão, Luiz } \\
\text { Jorge. }\end{array}$ & Lilacs & 2009 & \\
\hline
\end{tabular}

Verificou-se a existência de um número reduzido de estudos referentes ao tema proposto. Foi encontrado um total de 26 artigos, sendo que 4 deles estavam em triplicidade e 3 em duplicidade, restando exatos 15 artigos para análise. Estes foram categorizados da seguinte maneira: tratamento familiar (4 artigos), fatores de risco e proteção (4 artigos), adesão ao tratamento (1 artigo), drogas e violência (1 artigo), leis e políticas públicas (1 artigo), drogas e maternidade (1 artigo), rede social (1 artigo), saúde mental (2 artigos). Dentre as categorias apresentadas, apenas os 5 artigos categorizados em "tratamento familiar" abordam o objeto de estudo desta pesquisa. Porém, um deles (número 3) aborda o tratamento familiar no Uruguai, e como o foco deste estudo consiste na assistência às famílias brasileiras, este artigo não será incluído na análise. Apesar dos artigos pertencentes às categorias "fatores de risco e proteção" e "rede social" aproximarem-se do tema, não abordam o tratamento familiar em si como coadjuvante ao tratamento do dependente químico, sendo, portanto, excluídos desta análise. As demais categorias foram excluídas da análise por não se enquadrarem ao tema desta pesquisa.

Dentre os 3 artigos encontrados que abordam tratamentos realizados com famílias no Brasil, todos eles foram produzidos pela área da enfermagem. A partir da análise deste material, foi possível identificar a existência de alguns modelos de tratamento familiar focados em famílias de dependentes químicos: Grupos de Apoio, Grupo de Orientação Familiar, e Terapia Comunitária Integrativa.As intervenções familiares discutidas, apesar de partirem de diferentes perspectivas teóricas, mostraram-se ferramentas importantes na atenção ao dependente químico. 


\section{Discussão dos resultados}

Desde a Reforma Psiquiátrica brasileira os familiares dos pacientes de saúde mental vêm sendo chamados a assumirem uma parceria no tratamento realizado tanto pelos CAPS, quanto ambulatórios, residências terapêuticas e rede básica de saúde (Ministério da saúde, 2005). A família tem sido, desde então, colocada como a principal cuidadora do dependente químico, devendo atender às suas demandas subjetivas de afeto até então mantidas trancafiadas no sistema de internação hospitalar (Alvarez, Gomes, Oliveira \& Xavier, 2012; Loyola, Brands, Adlaf, Giesbrecht, Simich\& Wright, 2009; Matos et al, 2008). Apesar da indiscutível importância da família neste cenário, é reduzida a produção cientifica sobre intervenções familiares realizadas no âmbito nacional, como tratamento coadjuvante da dependência química.

A família é considerada uma referência fundamental na vida do ser humano em relação à saúde mental. Nela coexistem experiências de pertencimento, afeto bem como conflitos, solidão e sofrimento (Cardoso \& Féres-Carneiro, 2008; Rezende, 2001). Por este motivo, é importante o estudo da família como fator de risco e proteção à dependência química (Lemos, Antunes, Baptista, Tufik, Mello \& Formigoni, 2012; Silva, Guimarães \& Salles, 2014; Vargens \& Brands, 2009). Tem havido uma crescente preocupação com o papel da família no tratamento de dependentes químicos ao longo das últimas décadas (Rezende, 1997), bem como a necessidade de inclúi-la neste, apesar de não haver consenso acerca da melhor abordagem a ser adotada. Quanto a isso, o OBID (2015) refere que as intervenções psicoterápicas oferecidas no Brasil - individuais, grupais, familiares ou sociais - são baseadas em diversas abordagens teóricas, desde a psicanálise (a mais utilizada como modelo de tratamento para dependentes químicos) até técnicas cognitivo-comportamentais. Os poucos artigos encontrados na revisão da literatura nacional corroboram parte desta informação, apresentando todos apenas intervenções grupais, porém a partir de diferentes abordagens teóricas: intervenções baseadas na concepção de grupo terapêutico (Alvarez, Gomes, Oliveira \& Xavier, 2012), no método de terapias focadas na solução (Matos, Pinto \& Jorge, 2008) e baseadas na teoria da comunicação (Rocha, Sá, Braga, Ferreira \& Dias, 2013). Observa-se a necessidade de realização de mais estudos e desenvolvimento de programas efetivos de intervenção voltados especificamente à população brasileira e uma implementação efetiva.

Dentre as pesquisas encontradas, o Grupo de Apoio proposto por Alvarez, Gomes, Oliveira e Xavier (2012) mostra-se uma opção efetiva como tratamento coadjuvante na dependência química, a qual, além de ser considerada uma doença, é considerada um sério problema de saúde pública que necessita de ações em busca de estratégias tanto para tratamento como para promoção e prevenção de saúde de usuários e familiares (Delfini, Sato, Antoneli \& Guimarães, 2009). Trata-se de uma estratégia de cuidado, realizada dentro do CAPS-AD, voltada para assistência familiar realizado por profissionais de saúde, mais especificamente pela enfermagem, que deve ser habilitada em técnicas interpessoais de comunicação, relacionamento terapêutico e manejo grupal. Esta ferramenta baseia-se na descrição de grupo terapêutico (Loomis, 1979), cuja linha central é o trabalho de reabilitação que pode contemplar as instâncias social, psicológica e física, com ênfase no potencial grupal para ajuda interpessoal.

Nesta perspectiva, o Grupo de Apoio, que acontece em uma periodicidade pré-definida, deve poder auxiliar as pessoas a se ajustarem em períodos de mudanças, no tratamento de crises, bem como na manutenção ou adaptação a novas situações (Alvarez, Gomes, Oliveira \& Xavier, 2012). Segundo os autores, o Grupo de Apoio do CAPS AD possui objetivo terapêutico e auxilia no enfrentamento de momentos de crise. Além de elevar autoestima dos participantes, facilita à família servir de alicerce para o dependente químico durante sua recuperação. Também demonstra impactar de forma positiva na percepção da realidade e do problema vivido. Observaram ainda melhora no alívio de sentimentos de solidão e isolamento social vivido pelas famílias participantes, através da troca de experiências e reflexão. O Grupo de Apoio se mostra uma ferramenta efetiva para a realização de educação em saúde, prevenção, promoção e recuperação da saúde tanto de indivíduos como de grupos sociais, provocando nas famílias sentimentos de acolhimento, pertencimento e fortalecimento.

Outros estudos têm apontado que famílias que recebem o cuidado através de terapia familiar apresentam um melhor desfecho que aquelas não incluídas no tratamento (O'Farrel, 1992; Rezende, 2001; Stanton \& Shadish, 1997). Sabe-se também que cerca de $80 \%$ de pacientes dependentes químicos que utilizaram serviço ambulatorial aderiram melhor ao tratamento quando sua família também era atendida quando comparado aqueles cujas famílias não o eram (Figlie, Fontes, Moraes \& Paya, 2004). Tal ocorrência pode estar relacionada ao fato de que muitas vezes os membros da família ficam presos num padrão de comunicação ou atuação que impossibilitam a mudança, pois proporcionam, num nível inconsciente, 
gratificação a impulsos e fantasias que compensam a dor causada pela doença.

O tratamento familiar, mesmo que com intervenções breves, pode colaborar para desfazer este impasse de maneira a tornar novamente disponível as capacidades de cooperação ponderada de cada membro, colaborando para uma quebra deste padrão (Davison, 1997). Matos, Pinto e Jorge (2008) demonstraram que o Grupo de Orientação Familiar, realizado no Centro de Convivência para dependentes químicos de um hospital psiquiátrico público de Fortaleza, no Ceará, apresenta resultados positivos neste sentido.

O Grupo de Orientação Familiar (Matos, Pinto \& Jorge, 2008), baseia-se no método das terapias focadas na solução, cujo foco se coloca não em examinar as causas da doença, mas sim explorar as soluções para as dificuldades encontradas sem atribuir responsabilidades implícitas. Parte de um modelo cognitivo, e não exige neutralidade do terapeuta. Consiste num trabalho de orientação e sensibilização familiar com objetivo de melhorar, justamente, a qualidade das relações entre seus membros de forma a colaborar com a recuperação do paciente. Possui caráter informativo-educativo, buscando responder às demandas familiares em relação à dependência química. Observa-se impacto positivo nos sentimentos da família, na compreensão da dependência química enquanto patologia além de auxiliá-los no manejo com o familiar dependente.

Kalina (1998) chama atenção ao fato de que, usualmente, a família do dependente químico possui uma percepção distorcida de seus problemas, muitas vezes comuns, mas que ganham outra dimensão e intensidade, a ponto de tornar-se co-dependente. Não raro, ao mesmo tempo em que um membro da família desenvolve a dependência química, estabelece-se uma dinâmica na qual muitas vezes os familiares também passam a "necessitar" da droga, estruturando-se as relações de co-dependência (Filgie, Fontes, Moraes \& Paya, 2004; Rezende, 1997). Neste sentido, a Terapia Comunitária Integrativa (Rocha et al, 2013) busca auxiliar a família a encontrar soluções para os conflitos pessoais e familiares, através de uma estratégia de inclusão social e apoio à saúde mental da população por meio do oferecimento à família de um espaço de escuta, reflexão, troca de experiências e acolhimento do que emerge do grupo. Sustentada pela Teoria da Comunicação, parte do pressuposto de que a comunicação entre os indivíduos é o que os une, bem como à família e à sociedade. A teoria da comunicação refere que, para a compreensão do significado do que é comunicado, deve-se necessariamente levar em conta o comportamento do sujeito, a linguagem utilizada e a relação entre os que se comunicam (Rocha et al, 2013).

Rezende (1997) ressalta que a família do dependente químico tende a preocupar-se prioritariamente com os prejuízos morais, sociais e familiares do que os danos causados ao indivíduo. A Terapia Comunitária Integrativa possibilita uma percepção mais crítica da realidade vivida e das necessidades, bem como o desenvolvimento de potencialidades e a busca conjunta de meios para resolução de problemas, mostrando-se um instrumento que permite o desabafo e alivio de sentimentos, buscando promover a resiliência. Verificou-se, nas famílias participantes, melhora da autoestima, bem-estar, resiliência e diminuição de sentimentos angustiantes (Rocha et al, 2013).Porém, não demonstra atuar no material latente da dinâmica familiar, no qual muitas vezes a droga vem então servir como uma maneira de mascarar os reais problemas familiares, concentrando no dependente a fonte de todos os seus problemas e evitando crises que ameacem desestruturar a dinâmica estabelecida, sendo imprescindível tratar os aspectos psicodinâmicos tanto a família quanto o paciente para incidir na homeostase familiar patológica (Rezende, 1997, 2001; Stemschuss, 1982).

Nas pesquisas encontradas, há prevalência de estratégias grupais de intervenções terapêuticas com familiares de dependentes químicos. Eiguer (1985) refere a importância da terapia familiar se fazer através linguagem do grupo familiar como um todo, e de ocupar-se em estudar a dinâmica grupal-familiar regida por forças inconscientes. Dessa forma, pode auxiliar no desenvolvimento da autonomia dos psiquismos individuais de cada um dos membros familiares interpretando, por isso, as emoções, o amor conjugal, filial, fraterno e o funcionamento dos papéis sexuais presentes no seio familiar. Ressalta ainda que a terapia familiar deve buscar o ajustamento dos indivíduos no grupo familiar de tal forma que as relações se tornem menos tensas e mais compreensivas

Tais intervenções grupais, nas quais os participantes convivem com outros que vivenciam situação semelhante, facilitam a identificação, a intimidade e o apoio mútuo entre os membros do grupo. Observa-se nas estratégias de tratamento familiar encontradas, preocupação em acolher as famílias e atuarem como fonte de informação sobre a doença e de criação de estratégias que a fortaleçam e orientem no manejo com o dependente químico. Desta forma, ela terá melhores condições colaborar com a evolução do mesmo. Prevalece nas intervenções um caráter informativo, educativo, de orientação e apoio ao grupo familiar, respondendo desta forma à demanda manifesta 
pela família (Alvarez, Gomes, Oliveira \& Xavier, 2012; Matos, Pinto \& Jorge, 2008; Rocha et al, 2013).

As famílias atendidas demonstraram tendência a perceber a dependência química apenas como uma doença biológica, desconsiderando outros aspectos importantes envolvidos na questão, como os subjetivos. Os estudos demonstram a necessidade de revisão desta visão biológica da dependência química, em vistas da evidência de existência tanto de processos psicológicos (cognitivos e afetivos) quanto ambientais e sociais envolvidos neste quadro (Matos, Pinto \& Jorge, 2008; Rocha et al, 2013).

As famílias, por sua vez, apreciam os grupos interventivos, pois encontra ali um ambiente livre de julgamentos e críticas, um ambiente de acolhimento que diminui o sentimento de solidão e auxilia a compreender a doença e a situação vivenciada (Alvarez, Gomes, Oliveira \& Xavier, 2012; Matos, Pinto \& Jorge, 2008). Porém, costumam vincular-se ao tratamento familiar apenas enquanto o dependente se mantivesse em tratamento, evidenciando a co-dependência familiar na patologia (Matos, Pinto \& Jorge, 2008).

É inegável a importância da capacitação dos profissionais que realizam intervenções familiares no sentido de identificar em os fatores que interferem no uso de drogas pelo paciente (Rezende, 2001). Observa-se que no modelo de tratamento que conta com a presença do psicólogo - dentre os 3 modelos encontrados, apenas 1 o objetivo primeiro não está no âmbito do informativo ou educativo, mas de alívio de sofrimentos, de fortalecimento de vínculos, de auxiliar as famílias a desenvolverem percepção mais crítica da realidade e das necessidades vividas pela família. Prevalece a prioridade em oferecer a oportunidade de encontrarem estratégias de enfretamento mais adequadas e de construir um saber próprio, mesmo em grupo, a partir da história de cada família, buscando empoderá-las de suas próprias vidas (Rocha et al, 2013).

Não restam dúvidas de que o envolvimento e tratamento da família do dependente químico não apenas impacta de forma positiva em sua recuperação, como também no relacionamento familiar. Além disso, Rezende (2001) ressalta que estes cuidados facilitam a reinserção social, devendo a intervenção familiar estar incorporada aos programas de tratamento destes pacientes. Porém, observa-se uma limitação do Estado na oferta de serviços específicos, e poucas pesquisas realizadas no Brasil neste sentido. Varges e Brands (2009), relatam que os serviços mais encontrados referentes a tratamento para dependentes químicos e suas famílias são aqueles oferecidos pelas instituições religiosas $(51.5 \%)$ e grupos de autoajuda dirigida por ex-usuários $(27,2 \%)$ e que estes não recebem nenhum tipo de apoio do Estado. Além disso, mesmo nas entidades não governamentais, o acesso ao tratamento não é possível para todos, e familiares e usuários queixam-se da dificuldade de acesso aos serviços públicos (Loyola et al, 2009; Vargens \&Brands, 2009). Faz-se necessário então que a proteção e suporte a essas famílias seja alvo das políticas públicas (Alvarez, Gomes, Oliveira \& Xavier, 2012; Loyola, Brands, Adla, Giesbrecht, Simich\& Wright, 2009; Matos et al, 2008), e neste sentido, chama novamente a atenção a escassez de trabalhos encontrados, bem como da participação da Psicologia nestes.

\section{Considerações Finais}

Apesar do significativo impacto da dependência química na saúde pública do Brasil e da criação de dispositivos de atendimento que preveem não apenas o atendimento ao dependente, mas também de sua família, observa-se escassa produção de conhecimento científico visando o desenvolvimento de estratégias de tratamento familiar que sejam viáveis, acessíveis e não se limitem apenas a espaços grupais informativos, educativos e de acolhimento. É inegável a influência da família enquanto fator de risco ou proteção ao desenvolvimento da dependência química, bem como sua importância na evolução do tratamento ou duração da abstinência.

Por estes motivos, faz-se fundamental o desenvolvimento de estratégias interventivas que possam alcançar não apenas aquilo que se manifesta como demanda da família - como maior compreensão sobre a doença, orientações quanto o manejo do dependente no ambiente familiar, e alívio de sentimentos de angustia e sofrimento, por exemplo - que sem dúvida são fundamentais e impactam de forma positiva na família e em suas relações. Porém, não podem ser ignorados os aspectos subjetivos subjacentes, latentes, que permeiam a constituição dessas mesmas relações e atuam na manutenção e na repetição do sintoma. Para isso, são necessárias pesquisas desenvolvidas na área da psicologia que desenvolvam e avaliem estratégias que possam impactar nestes aspectos.

Partindo do princípio que a família tem como função primordial a proteção de seus membros, tendo potencial para oferecer apoio emocional para resolução de problemas e conflitos, cabe aos profissionais que lidam diretamente com as questões da dependência química orientar e estimular os familiares, fortalecendo vínculos e facilitando o processo que envolve o tratamento do dependente. Faz-se necessário que os serviços tenham 
como rotina avaliar forças e necessidades da dinâmica familiar dos dependentes, assim como avaliar o impacto da dependência na família ou contexto social, considerando os membros da família afetados. Fundamental a realização de avaliações frequentes, quantitativas e qualitativas, dos resultados das intervenções realizadas nas diversas modalidades de serviços oferecidos, a fim de que se aprimorem projetos visando melhor eficácia para atender demandas de diferentes contextos socioculturais.

\section{Referências}

Abdalla, R.R., Madruga, C.S., Ribeiro, M., Pinsky, I., Caetano, R., \& Laranjeira R. (2014). Prevalence of Cocaine Use in Brazil: Data fromthe II BrazilianNationalAlcoholandDrugsSurvey (BNADS). Addict Behav, 39, 297-301. Recuperado de https://www.ncbi.nlm.nih.gov/pubmed/24455783.

Aguilar, L.R., \&Pillon, S.C. (2005). Percepción de tentaciones de uso de drogas en personas que recibentratamiento. Revista Latino-Americana de Enferm.;13(spe), 790-797. Recuperado de https://dx.doi.org/10.1590/ S0104-11692005000700005

Alvarez, S.Q.,Gomes, G.C., Oliveira, A.M.N., \&Xavier, D.M. (2012). Support group as a strategy of care: the importance for relatives of drug users. Revista Gaucha de Enfermagem, 33(2), 102-108. Recuperado de https://dx.doi. org/10.1590/S1983-14472012000200015

Brasil. Ministério da Saúde (2007). Secretaria de Atenção à Saúde.Relatório de Gestão 2003-2006: saúde mental no SUS: acesso ao tratamento e mudança do modelo de atenção. Brasília: Ministério da Saúde.

Brasil. Ministério da Saúde. (2005). Secretaria de Atenção à Saúde. DAPE. Coordenação Geral de Saúde Mental. Reforma psiquiátrica e política de saúde mental no Brasil. Documento apresentado à Conferência Regional de Reforma dos Serviços de Saúde Mental: 15 anos depois de Caracas. OPAS. Brasília: Ministério da Saúde.

Brasil. Ministério da Saúde. (2004). Secretaria de Atenção à Saúde. SVS/CN-DST/AIDS. A política do Ministério da Saúde para Atenção Integral a Usuários de Álcool e Outras Drogas. Brasília: Ministério da Saúde.

Canoletti, B., \& Soares, C.B. (2005). Programas de prevenção ao consumo de drogas no Brasil: uma análise da produção científica de 1991 a 2001. Interface Comunicação, Saúde, Educação, 9(16), 115-29. Recuperado de https:// dx.doi.org/10.1590/S1414-32832005000100010

Cardoso, C.L., \& Féres-Carneiro, T.F. (2008). Sobre a família: com a palavra, a comunidade. Estudos e Pesquisa em Psicologia, 8(2), 511-526. Recuperado de http://pepsic.bvsalud.org/pdf/epp/v8n2/v8n2a25.pdf.

Davison, S. (1997). Porque alguns precisam mais e outros menos de tratamento. In: Edwards, G., \&Dare,C. Psicoterapia e tratamento de adições. Porto Alegre, RS: Artes Médicas.

Delfini, P.S.S., Sato, M.T., Antoneli, P.P., \& Guimarães, P.O.S. (2009). Parceria entre CAPS e PSF: o desafio da construção de um novo saber. Ciências e Saúde Coletiva, 14(1), 1483-92. Recuperado de http://www.scielo.br/pdf/ $\mathrm{csc} / \mathrm{v} 14 \mathrm{~s} 1 / \mathrm{a} 21 \mathrm{v} 14 \mathrm{~s} 1$

Eiguer, A. (1985). Um divã para a família. Porto Alegre, RS: Artes Médicas.

Figlie, N., Fontes, A., Moraes, E.,\&Paya, R. (2004). Filhos de dependentes químicos com fatores de risco bio-psicossociais: necessitam de um olhar especial? Revista de Psiquiatria Clínica, 31(2). Recuperado de http://www. scielo.br/scielo.php? script=sci_arttext\&pid=S0101-60832004000200001\&ln $\mathrm{g}=\mathrm{en} \& \mathrm{nrm}=$ isso

Kalina, E. (1988). Drogadição II. Rio de Janeiro, RJ: Francisco Alves.

Lemos, V.A., Antunes, H.K.M.,Baptista, M.N.,Tufik, S.,Mello, M.T., \&Formigoni, M.L.O.S. (2012).Low family support perception: a 'social marker' of substance dependence? Revista Brasileira de Psiquiatria, 34(1), 52-59. Recuperado de https://dx.doi.org/10.1590/S1516-44462012000100010

Loomis, M.E. (1979). Group process for nurses. Saint Louis: Mosby Company.

Loyola, C.M.D., Brands, B.,Adlaf, E., Giesbrecht, N.,Simich, L.,\&Wright, M.G.M.(2009). Illicit drug use and the critical perspectives of drug users' relatives and acquaintances in Northern Rio de Janeiro (City), Brazil.Revista Latino Americana de Enfermagem, 17(spe), 817-823. Recuperadode https://dx.doi. org/10.1590/S0104-11692009000700010

Matos, M.T.S.,Pinto, F.J.M.,\&Jorge, M.S.B. (2008). Family orientation in chemical dependence group: an evaluation on family members perception. Revista Baianade Saúde pública, 32(1), 58-71. Recuperado dehttp://inseer.ibict.br/rbsp/ index.php/rbsp/article/viewFile/1384/1020

Observatório Brasileiro de Informações sobre Drogas - OBID. (2015). Tratamento/ Dependência Quimica /Diferentes Abordagens. Recuperado de: http:// www.obid.senad.gov.br/portais/OBID/conteudo/index.php?id_c10onteu $\mathrm{do}=11417$ \&rastro $=$ TRATAMENTO $\% 2$ FDepend $\% \mathrm{C} 3 \%$ AAncia + Qu $\%$ C3 $\%$ ADmica/Diferentes+abordagens.

O'Farrel, T.J. (1992). Families and alcohol problems: An overview of treatment research. Journal of Family Psychology, 5, 339-59. Recuperado de http://psycnet.apa.org/index.cfm? fa=buy.optionToBuy\&id=1993-03092-001

Organização das Nações Unidas - ONU. (2015). Departamento de Drogas e Crimes, Recuperado dehttp://nacoesunidas.org/cerca-de-246-milhoes-de-pessoas-usaram-drogas-ilicitas-em-2013-afirma-novo-relatorio-da-onu/

Rezende, M.M. (1997). Curto-circuito familiar e Drogas. Taubaté, SP: Cabral Editora Universitária.

Rezende, M.M. (2001). Abordagem familiar no tratamento de toxicodependentes: aspectos esquemáticos de uma modalidade de atendimento. Psicólogo informação, 5(5), 35-44.

Rocha, J.A.,Sá, A.N.P.,Braga, L.A.V.,Ferreira Filha, M.O., \&Dias MD. (2013). Community integrative therapy: situations of emotional suffering and patients' coping strategies. Revista Gaucha de Enfermagem, 34(3), 155-162. Recuperadode https://dx.doi.org/10.1590/S1983-14472013000300020

Silva, M.L.,Guimarães, C.F.,\&Salles, D.B. (2014). Risk and protective factors to prevent relapses of psychoactive substances users.Revista RENE, 15(6), 1007-101. DOI: 10.15253/2175-6783.2014000600014

Stanton, M.D., \& Shadish, W.R. (1997). Outcome, attrition, and family/couples treatment for drug abuse: a meta-analyses and a review of controlled, comparative studies. PsychologicalBulletin, 122, 170-91. Recuperado de http:// psycnet.apa.org/index.cfm? fa=buy.option'ToBuy\&id=1997-05606-004

Sternschuss, S. (1982). A família do toxicômano. In: Olievenstein, C. A Vida do Toxicomano. Rio de Janeiro, RJ: Zahae.

Vargens, O.M.C., \&Brands, B.(2009).Use of illicit drugs and critical perspectives of drug users' relatives and acquaintances in Northern Rio de Janeiro (City), Brazil.Revista Latino Americana de Enfermagem, 17(spe), 776-782. Recuperado de https://dx.doi.org/10.1590/S0104-11692009000700004

Submetido em: 13-12-2016

Aceito em: 6-3-2017 